\title{
Agro asociativo, lucha por la tierra y comunidad después de la reforma agraria en Puno, Perú ${ }^{1}$
}

\section{Associative agriculture, the struggle for land and community after the agrarian reform in Puno, Peru}

María Luisa Burneo ${ }^{2}$

Instituto de Estudios Peruanos

\section{Resumen}

El artículo traza las trayectorias que siguieron las empresas asociativas en Puno después de la reforma agraria. Analiza los cambios en la estructura de la tierra a la luz de los procesos de reestructuración agraria y de la formación de nuevas comunidades campesinas. El planteamiento central es que la reforma agraria implementada por el gobierno de Velasco tuvo particularidades que deben ser incorporadas en la lectura de esta como un proceso con variaciones regionales. Asimismo,

1 Este artículo es producto de la investigación Agro asociativo y procesos sociales: Transformaciones rurales desde la reforma agraria en la sierra centro y sur peruana, desarrollada con la dirección del profesor Alejandro Diez Hurtado y el apoyo del Instituto Riva Agüero, de la Pontificia Universidad Católica del Perú.

2 Magíster en Antropología Social y Etnología por la Escuela de Altos Estudios de Ciencias Sociales (París, Francia) y licenciada en Antropología por la Pontifica Universidad Católica del Perú. Desde hace veinte años, trabaja sobre los procesos de cambio en las sociedades rurales, referidos particularmente a las comunidades campesinas, la política local y la gestión de los recursos naturales. Código ORCID: 0000-0002-3212-2921. Contacto: mburneo@iep.org.pe 
se sostiene, a partir del análisis y trayectoria de dos grandes empresas asociativas -Picotani y Kunurana-, que el aparato del gobierno velasquista no realizó una lectura adecuada de las distintas lógicas de tenencia de la tierra de las familias campesinas ganaderas, ni de la presión de las comunidades colindantes por los recursos de agua y tierra. La lucha por la tierra, surgida por las expectativas de miles de familias comuneras y por una distribución considerada desigual, es uno de los ejes centrales de este texto. Desde esa perspectiva, la reforma agraria, más que un evento que duró unos años (1969-1975), fue un proceso que se superpuso a dinámicas previas de más largo plazo y se insertó en territorios diversos y en microprocesos sociales en curso con los cuales se traslapó, lo que generó tensiones y conflictos en las siguientes dos décadas. En el caso que se ha estudiado, ello implicó la reconfiguración del espacio surandino y la consolidación de cientos de comunidades con un régimen de tenencia de la tierra parcelario en su interior.

Palabras clave: reforma agraria, apropiación de tierras, agro asociativo, movimientos campesinos, sociedades agrícolas de interés social, SAIS, comunidades campesinas, Puno, Perú

\section{Abstract}

The article traces the trajectories followed by associative enterprises after the agrarian reform in Puno. It analyzes the changes in the structure of the land in light of the processes of agrarian restructuring and the formation of new peasant communities. The central argument is that the agrarian reform implemented by the Velasco government had peculiarities that should be incorporated in the reading of it as a process with regional variations. Likewise, it is argued, based on the analysis and history of two large associative companies -Picotani and Kunurana- that the Velasquista government's 
apparatus did not make an adequate reading of the different land tenure logics of the peasant livestock families, nor of the pressure for water and land resources of the surrounding communities. The struggle for land, which arose from the expectations of thousands of communal families and from a distribution considered unequal, is one of the central axes of this text. From this perspective, the reform, more than an event that lasted from 1969 to 1975 , was a process that was superimposed on previous, longer-term dynamics, which was inserted in diverse territories and in ongoing social microprocesses with which it overlapped, generating tensions and conflicts in the two following decades. In the case studied, this implied the reconfiguration of the South Andean space and the consolidation of hundreds of communities with a land tenure regime within it.

Keywords: agrarian reform, land appropriation, associative agriculture, peasant movements, agricultural societies of social interest, SAIS, peasant communities, Puno, Perú

\section{Introducción}

Se ha tendido a interpretar la reforma agraria peruana como un evento radical por la cantidad de fundos expropiados y tierras afectadas (Eguren, 1988 y 2004), que significó, sin duda, un profundo cambio en la sociedad rural peruana. Este cambio se ha discutido, principalmente, en términos de éxito o fracaso y en función de la manera en que el gobierno velasquista la implementó, imponiendo lo que se podría llamar el modelo del agro asociativo (Caballero, V. 1990; Caballero y Álvarez 1980). Sin embargo, no se ha relevado la importancia de los procesos regionales y locales que hicieron que esta reforma tomara caminos distintos. Como ha señalado Enrique Mayer, 
hace falta comprender que también se hizo una reforma "desde abajo" " ${ }^{3}$ En el caso de Puno, ámbito del presente artículo, la presencia del movimiento campesino y su búsqueda de una mejor redistribución de la tierra expropiada a través de las tomas de tierra fueron el motor de la reestructuración agraria (Caballero, 1990; Rénique, 2004; Scurrah, 1987). Al igual que este caso, en otros lugares del Perú el aparato velasquista también encontró demandas locales previas que, si bien aplaudían la expropiación de las haciendas, estuvieron en desacuerdo con el modelo del agro asociativo (Diez y Burneo, 2019) e incluso propusieron alternativas comunales propias (Burneo, 2019; Diez, 2017; Van Der Ploeg, 2018).

Partiendo de lo anterior, se propone una lectura de la reforma agraria como un proceso de trayectorias regionales diferenciadas, que tomó formas particulares en función de las distintas lógicas de tenencia de la tierra y dinámicas comunales previas. Para ello, se desarrolla el proceso del agro reformado en Puno, un proceso de luchas y reestructuraciones no saneadas, que dejaron espacio para el surgimiento de nuevas formas de organización y la consolidación de la vía comunera. Cabe anotar, que esa vía comunera se dio también en otros ámbitos regionales, como en el caso de Junín, pero con las particularidades de un espacio dinámico y en transformación, con mayor articulación al mercado (Long y Roberts, 1978). En este texto, se aborda el caso del sur andino, Puno en particular, que presenta una trayectoria caracterizada por la búsqueda de las familias campesinas y comuneras al acceso y control directo de la tierra, que durante décadas estuvo bajo el poder de las haciendas.

3 Sesión de debate con Enrique Mayer organizada por el Taller Etnológico de Cultura Política de la Pontificia Universidad Católica del Perú en el año 2017 con la conducción de la autora y del profesor Alejandro Diez Hurtado. 
Se desarrolla la idea propuesta en el párrafo anterior a partir del análisis de las trayectorias de dos empresas asociativas de zonas representativas de la reestructuración agraria ocurrida luego de la implementación de la reforma. La primera, la SAIS Picotani en el distrito de Macusani, provincia de Carabaya, y su proceso de fragmentación, el cual dio origen a las comunidades campesinas Culloc, Belén y Chuquini. La segunda, la Empresa Rural de Producción Social (ERPS) Kunurana en el distrito de Melgar, ámbito emblemático de las tomas de tierra ${ }^{4}$. Los hallazgos del análisis realizado permiten sostener que la reforma agraria, si bien impuso el modelo asociativo, generó un conjunto de procesos con variantes regionales que se explican por las estructuras de la propiedad, las formas de tenencia de la tierra y la lógica de la apropiación espacial previas, además de los ya estudiados problemas de gestión de las cooperativas y las SAIS que se repiten en diferentes zonas del país (Caballero, 1990; Scurrah, 1987). Esta superposición de lógicas y las brechas entre el modelo velasquista y las expectativas (y necesidades) de las familias campesinas son el eje de la discusión del presente texto.

El artículo está organizado en tres partes. La primera, desarrolla el contexto regional puneño -referido a la propiedad y formas de tenencia- sobre el que se implementó la reforma

4 El material recolectado para estos casos proviene de los archivos de la ex Dirección Regional de Reforma Agraria de Puno, los archivos de la Dirección Regional Agraria de Puno, así como de los informes inéditos del estudio Reforma y reestructuración agraria dirigido por José María Caballero en 1978, actualmente en custodia del Archivo Documental del Instituto del Perú, de la Universidad San Martín de Porres. Expreso mi agradecimiento a Giovanni Bonfiglio, investigador de dicho instituto, por facilitarnos el material referido. La información se ha complementado con entrevistas en profundidad realizadas en agosto de 2018 en Puno a exdirigentes campesinos y exfuncionarios de la reforma agraria. A todos ellos, mi sincero agradecimiento. 
agraria en Puno. La segunda, trata el problema de la presión interna por los pastos y presenta información que permite dar cuenta de las trayectorias posteriores a la reforma y sus tensiones en el interior de las empresas asociativas. La tercera y última parte analiza la presión de las comunidades colindantes, así como las razones de las tomas de tierra lideradas por las comunidades indígenas de Melgar, con existencia previa a la reforma, y el proceso de formación de nuevas comunidades campesinas.

\section{La reforma agraria en Puno: gamonalismo, despojo y ex- pectativa por la tierra}

Antes de la reforma agraria, Puno presentaba un escenario caracterizado por la presencia de un número importante de haciendas que podrían definirse como "tradicionales", en las que imperaba el régimen del gamonalismo (Bourricaud, 1967; Gascón, 1999 y 2017; Jacobsen, 2013; Matos Mar y Mejía, 1980), y al mismo tiempo por el avance de un movimiento campesino que sin alcanzar una articulación departamental estaba tomando forma en diversas localidades, como en la zona de Melgar (Rénique, 2004). El gamonalismo es el régimen asociado a este tipo de haciendas, que albergaban a la población indígena y campesina en situación de explotación y condiciones inhumanas bajo la forma del pongaje (Matos Mar y Mejía, 1980; Mayer, 2009). Para las comunidades circundantes a las haciendas, así como para los feudatarios de estas últimas, el problema central era el acceso a la tierra. Además, a diferencia de otras zonas del país, en donde existían haciendas con un régimen asalariado y trabajadores permanentes y eventuales, en el sur andino, el pongo pertenecía al universo de la hacienda, debía prestar servicios al gamonal más allá de su mano de obra agrícola o pastoril, y era sometido -junto con su familia- a servicios personales, al despojo de sus tierras y 
ganado, así como a castigos físicos 5 . No es por azar que haya sido en Puno donde se gestaron, varias décadas antes, importantes levantamientos y rebeliones contra la explotación de los gamonales, como la de Juan Bustamante (1867) o la de Rumi Maqui (1915). Gascón (1999) retrata, para el caso de Amantaní en el mismo departamento, el sistema de explotación de los indígenas en el interior de la hacienda y resalta la dependencia del gamonal para su sobrevivencia, así como el control casi absoluto que este tenía de la administración de la justicia local, como parte de un pacto implícito con el Estado (Gascón, 2017).

Tratándose de zonas altas, entre los 3800 y los 4300 m.s.n.m., la actividad ganadera era fundamental y las familias feudatarias poseían su propio ganado huaccho o criollo, para lo cual requerían extensiones de pasturas considerables. El régimen de apropiación espacial de las sociedades pastoriles es de alta movilidad, por lo que no es suficiente contar con un área pequeńa de pastoreo, sino que se requieren varias zonas dentro de un territorio amplio que les permita el acceso a bofedales y un manejo rotativo de los pastos (Damonte y Rodríguez, 2016). Para ello, existían acuerdos de uso y ocupación del espacio de la puna, que funcionaban en el interior de la hacienda (y sus colonos) y entre esta y las comunidades colindantes. Este pequeño ejemplo de los pastores de hacienda o huacchilleros señala que el mundo de las haciendas era menos homogéneo y estático de lo que en ocasiones se asume en el imaginario colectivo respecto a su funcionamiento en las zonas alto andinas. Una combinación de relaciones de explotación, que diversos autores han clasificado como servil (Bourricaud, 1967; Favre, 1976), coexistía con arreglos informales, pero

5 El documental Agripino, del cineasta sueco Jan Lindqvisk (1971), recoge testimonios de primera mano sobre estas formas de explotación en la sierra sur del departamento de Cusco. 
institucionalizados a lo largo de generaciones sobre el uso del espacio entre los pastores de puna, la hacienda y las comunidades (Del Pozo-Vergnes, 2004).

Otra de las características del espacio del altiplano puneño antes de la reforma agraria es que se trataba de un territorio con importantes comunidades y parcialidades indígenas, que en 1969 eran 486 y 500, respectivamente. A lo largo de décadas, muchas de estas redujeron sus territorios por el avance de las haciendas. Sin embargo, luego de que el gobierno revolucionario expropiara 1450 fundos en Puno, solo 76 comunidades se convirtieron en adjudicatarias directas de la reforma agraria (Vega, 2005). El modelo del agro reformado, por tanto, no contentó a miles de familias que esperaban el acceso directo a la tierra. En respuesta, las familias campesinas y las comunidades jugaron un rol central en los años posteriores de la reforma y en las trayectorias que tomaron las empresas asociativas y sus socios.

En Puno, el proceso de implementación de la reforma agraria dio origen a 23 sociedades agrarias de interés social (SAIS), 5 empresas rurales de propiedad social (ERPS) y 14 cooperativas agrarias de producción (CAP) (Vega, 2005). Puno era uno de los departamentos con las haciendas ganaderas más grandes del país, por lo que las extensiones de pastura abarcaban varios miles de hectáreas. Algunas de estas habían logrado un nivel de tecnificación considerable para el procesamiento de la fibra de alpaca (Bourricaud, 346 1962). A pesar de ello, no se había producido una transferencia importante de tecnología hacia las comunidades, a diferencia de otras regiones, como en el centro del país, que sí se beneficiaron con el desarrollo tecnológico de las haciendas más modernas (Long y Roberts, 1978). En las haciendas puneñas existían diversos sistemas de tenencia de la tierra; se conocen casos en los que las tierras de los indios continuaban siendo manejadas con una lógica parcelaria previa a la conformación de la hacienda misma (Del Pozo- 
Vergnes, 2004). Cuando llegó la reforma agraria a Puno, la formación de las grandes empresas asociativas implicó la integración o concentración parcelaria, que reunió bajo el modelo cooperativista a miles de familias campesinas que conducían sus tierras individualmente, muchas de las cuales provenían de antiguas parcialidades indígenas y habían sido absorbidas dentro del territorio de las haciendas años atrás. Es decir, las grandes empresas del agro reformado se formaron sobre las bases productivas de las exhaciendas, además de las familias feudatarias (y sus lógicas previas), y también se anexaron e integraron a las comunidades de la zona. Otras se resistieron y quedaron en áreas colindantes.

La implementación de la reforma agraria fue a paso lento en el caso de Puno. Recién en 1973 se iniciaron las afectaciones y se llegó a expropiar un área de 2094479 hectáreas, de las cuales el $88 \%$ pasó a manos de las empresas asociativas. A partir de 1980, diversos sucesos en Puno aceleraron la liquidación de las SAIS y las ERPS, como se describe en los casos analizados de la sección siguiente. En las entrevistas realizadas durante esta investigación, exfuncionarios de la reforma agraria en Puno se refieren a problemas internos de gestión y corrupción en los propios equipos técnicos del Sistema Nacional de Apoyo a la Movilización Social (SINAMOS) y de los consejos de vigilancia y administración de las empresas asociativas. Como relata Juan Palao, alto funcionario de la reforma agraria en Puno, el descontento entre los socios beneficiarios y campesinos había crecido tanto en la zona norte como en el sur de la región ${ }^{6}$. Sin embargo, también se identificaron otras variables que explican ese descontento, las cuales se vinculan con el acceso a la tierra. Así, por ejemplo, Palao señala que la ley de cooperativas solo permitía que los hijos residentes heredasen la tierra en condición de socios.

6 Exjefe de la Oficina Nacional de Desarrollo Cooperativo (ONDECOP) Puno, durante la implementación de la reforma agraria. 
Por tanto, quienes querían enviar a sus hijos a estudiar fuera, arriesgaban su herencia futura, ya que las tierras revertirían a la empresa. Esto habría sido uno de los factores que incentivaron a las familias feudatarias de las empresas asociativas a presionar por la parcelación, ya que querían asegurar el acceso y control de la tierra para las generaciones futuras ${ }^{7}$. Sin embargo, existía otro factor importante: la presión de las comunidades colindantes, que no habían sido beneficiarias directas de las adjudicaciones de tierras.

A través de los archivos revisados y las entrevistas, se han identificado los siguientes procesos posteriores a la reforma agraria en Puno: (i) parcelación de las empresas asociativas -CAP, SAIS y ERPS-; (ii) presión interna por parte de las comunidades socias (anexadas); (iii) demandas de las comunidades colindantes de las empresas y conflictos por tierras; (iv) demandas de reestructuración llevadas a la acción a través de las tomas de tierra; (v) repartos de tierras a comunidades y grupos campesinos; (vi) formación de nuevas comunidades campesinas; y (vii) inscripción y registro formal de comunidades campesinas (a partir de los dos procesos anteriores). A continuación, se analizan los casos de las dos grandes empresas asociativas elegidas, los que ayudarán a graficar los procesos señalados y a comprender las trayectorias posteriores a la reforma que originaron la actual estructura de propiedad parcelaria y comunera en el sur andino.

\section{El asedio interno: lógicas agropastoriles diferenciadas y la presión sobre los recursos}

Según las cifras de los informes inéditos del equipo dirigido por José María Caballero (1978), que recogió información

7 Entrevistas realizadas a exfuncionarios de la reforma agraria y a exdirigentes campesinos de Puno. 
de todas las SAIS a nivel nacional, uno de los problemas centrales en el sur andino era el uso de los pastos ${ }^{8}$. La SAIS Picotani había sido conformada sobre la base de 14 fundos expropiados y sumaba un total de 94940 hectáreas, que se extendían en más de cuatro distritos (Azángaro, Putina, Muñina y Huancané). En el cuadro 1, se observa que la mayor extensión de la SAIS correspondía a pastos naturales, salvo la avena forrajera para el ganado que apenas ocupaba 33 hectáreas. Es importante comprender que esas cantidades no incluían a las comunidades anexadas a la empresa como socias; es decir, esas tierras eran administradas a través de la gerencia para uso del ganado de la SAIS y de los exfeudatarios de la hacienda, los socios actuales. Este punto es fundamental para comprender las trayectorias y tensiones posteriores.

Luego de las adjudicaciones de las tierras expropiadas a las haciendas de la zona, la SAIS Picotani quedó conformada en 1974 por diez fundos y cuatro comunidades: Huayllapata, Uyuni, Pistuni y Quilca Puncu. El ganado de la hacienda comprendía 79508 cabezas, mientras que los socios tenían

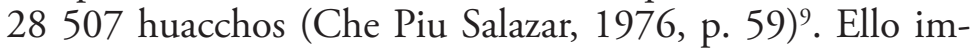
plica que cuando se creó la SAIS, el ganado de la empresa y de los exfeudatarios sumaban más de cien mil cabezas, principalmente ovinos. La presión que ello generaba sobre las pasturas era grande y a ello se añadió la presión por pastos

8 Informes técnicos de la ERPS Kunurana y de la SAIS Picotani sistematizados por José María Caballero (1978); actualmente custodiados por el Instituto del Perú de la Universidad San Martín de Porres.

9 Se denominaba huaccho al ganado de baja calidad, propiedad de los pastores en el ámbito de las haciendas ganaderas del sur andino. Por lo general, los huacchilleros (pastores de hacienda) pastaban su propio ganado en el interior de la hacienda, por lo que se les solía descontar un pago mensual de sus salarios; este pago, en ocasiones, equivalía al total del salario (Del Pozo-Vergnes, 2004). 
de las comunidades incorporadas a la SAIS, cuyas familias miembro consideraban que no contaban con suficiente tierra y que la distribución de las tierras expropiadas debió considerarlas como beneficiarias directas. Así, el descontento por el acceso a las pasturas se sintió desde muy temprano en las nuevas empresas asociativas.

\section{Cuadro 1. Distribución de terrenos según la capacidad receptiva SAIS Picotani, 1978}

\begin{tabular}{|c|l|c|}
\hline $\begin{array}{c}\text { Extensión en } \\
\text { Has. }\end{array}$ & $\begin{array}{c}\text { Pastos naturales según } \\
\text { tipo }\end{array}$ & $\begin{array}{c}\text { Capacidad Ov. a } \\
\text { Red. }\end{array}$ \\
\hline 500 & $1^{\circ}$ clase x 4.0 Ov. Red. & 2,000 \\
\hline 13,628 & $2^{\circ}$ clase x 3.0 “ “ & 40,884 \\
\hline 59,052 & $3^{\circ}$ clase x 2.0 “ “ & $1,128,104$ \\
\hline 16,675 & $4^{\circ}$ clase x 1.0 “ “ & 16,675 \\
\hline 1,438 & $5^{\circ}$ clase x 0.5. “ “ & 719 \\
\hline 33 & $\begin{array}{l}\text { Avena forrajera x 25 Ov. } \\
\text { Red. }\end{array}$ & 825 \\
\hline 45 & Cultivos anuales & ----- \\
\hline $3,569.44$ & Terrenos marginales & ------ \\
\hline $94,940.44$ & Has. Totales & 179,207 \\
\hline
\end{tabular}

Fuente: Informe de la SAIS Picotani. Informes de José M. Caballero (1978). Elaboración: José M. Caballero

350 Como se ha señalado, Picotani agrupaba a feudatarios (diversos entre sí) y a comunidades con lógicas propias de tenencia de la tierra que por lo general combinaban zonas de parcelas familiares, zonas de rotación reguladas por la comunidad (aynokas) y pasturas de uso colectivo. En el caso de los huacchilleros, estos habían forjado a lo largo de ańos sus propios acuerdos con la hacienda, los que les permitía acceder a distintas zonas de pastoreo en diversos momentos del año. Con lo anterior, Picotani era una 
mezcla de exfeudatarios y comunidades socias, con lógicas de producción diferenciadas y sistemas de tenencia de la tierra distintos. La idea central que se desprende de lo anterior es que más allá de los problemas de gestión de la empresa, en el interior de la misma y en la práctica ya existían las condiciones para su posterior fragmentación. Así, por ejemplo, el informe técnico elaborado para el IDISAPuno en 1976 registraba la falta de control respecto al uso de tierras en la SAIS. El informe señala que en los nuevos fundos incorporados a la empresa en las zonas colindantes a la antigua hacienda, los campesinos se apropiaban de parcelas de tierra a su antojo: "cuando tienen un ingreso extra, las siembran [las áreas anexadas] sin consultar a la administración central" (Instituto de Investigaciones Sociales del Altiplano, 1976, pp. 60-61).

Lo anterior indica que la superposición de lógicas de tenencia de la tierra y uso de pastos sumada a la presión de las comunidades colindantes anunciaban desde el inicio un proyecto sin cohesión interna, social y culturalmente difícil de consolidar. Más allá de los temas financieros y administrativos de la empresa, interesa mostrar las tensiones sociales por el acceso a la tierra vinculadas a la presión sobre lo pastos y las inconsistencias internas que influyeron en las trayectorias de los socios en los años siguientes. Así como se daba un uso al suelo que escapaba de la planificación de la empresa -y que más bien respondía a la lógica parcelaria de agricultura de autoconsumo de las familias campesinas-, también ocurría que los pastores se negaban a ir reduciendo su ganado huaccho en beneficio del ganado de la empresa y lo seguían manteniendo.

De los expedientes de adjudicaciones se desprende otro tema que se debe tomar en cuenta. Y es que para el aparato de la reforma velasquista, el ganado huaccho no solo era un problema productivo - puesto que dificultaba la rotación y el respeto 
a la nueva zonificación de pastos-, sino que también era un problema ideológico, indispensable de erradicar. Sucede que el sistema de acceso a pasturas de los huacchilleros se mantuvo durante décadas mediante acuerdos con la hacienda; por ejemplo, a cambio de la entrega de la fuerza de trabajo familiar -no solo del jefe de familia, sino de las mujeres y nińos- para cuidar y pastear el ganado del hacendado, este permitía que el ganado huaccho pastara por algunas zonas de la hacienda. También ocurría, cuando existía un salario, que se les descontaba un pago por el acceso a las pasturas. Los pastores que habían logrado acumular mayor cantidad de cabezas de ganado reprodujeron este sistema con los pastores más pobres o con menos tiempo en la hacienda. Este arreglo sobrevivió a la reforma agraria y continuó de manera informal en las SAIS recientemente conformadas. Para los funcionarios del gobierno, esta modalidad constituía el nodo de las relaciones serviles y de la "explotación del campesino por el campesino" y no debía permitirse. En efecto, los expedientes técnicos de la Dirección Regional Agraria (DRA) de la época evidencian la posición y preocupación de los funcionarios sobre el tema. Frente a ello, estos decidieron implementar una estrategia de capacitación de los campesinos y asumieron que los huacchilleros irían reduciendo su ganado huaccho al verse involucrados en la lógica capitalista de la empresa. Asumieron, también, que al ir constatando que el ganado de la empresa, de mejor calidad, obtenía mejores ganancias en el mercado, y al tener todos los socios salarios más altos gracias a ello, se iría reduciendo el ganado huaccho en beneficio de las ganancias colectivas. Sin embargo, cometieron un grave error.

Lo anterior permite señalar que, al parecer, el aparato técnico del gobierno y sus asesores tuvieron dificultad para comprender las lógicas pastoriles y las expectativas de la familias campesinas y ganaderas. Es posible hallar fragmentos de citas que resumen el pensamiento y la apuesta de los funcionarios; según ellos, eliminar el ganado huaccho era la acción im- 
prescindible para "acabar con las relaciones serviles y lograr la capitalización de la empresa” (Che Piu Salazar, 1976, p. 63). Pocos años después, la realidad mostró que las lógicas comunales y las dinámicas sociales de apropiación y uso del espacio tuvieron mayor peso que la apuesta por la empresa asociativa manejada por un equipo de técnicos y por pocos socios, en los cuales los exfeudatarios no confiaban. Y si estos no lo hacían, menos aún lo harían las familias de las comunidades anexadas.

En efecto, en lugar de reducirse, en 1978, los informes recuperados por el equipo de José María Caballero dan cuenta de que el ganado de la empresa superaba los 70 mil animales (había 55 mil ovinos, 14550 auquénidos y 1800 vacunos), mientras que el ganado huaccho de ovinos sumaban 45 mil unidades. Los exhuacchilleros de hacienda, para entonces socios de la SAIS, seguían manteniendo su propio ganado y sus sistemas de utilización de pastos en las zonas altas, sin apostar necesariamente por el modelo asociativo. Los informes económicos de la Dirección Regional de Reforma Agraria de Puno muestran que Picotani no estaba en pérdida en 1978; los egresos totales de la empresa sumaban poco más de veinticuatro millones de soles, mientras que los ingresos netos daban un total de 33000 210 soles; esto quiere decir en 1978, la SAIS habría tenido una ganancia de 8000993 soles. No existen informes económicos exhaustivos de los años previos, por lo que José María Caballero y su equipo concluyen que no es posible determinar si Picotani avizoraba un crecimiento sostenible o si iba hacia su descapitalización. Sin embargo, aunque no está formulado de esta manera, los hallazgos de Caballero del año 1978 permiten esbozar la idea de que los factores de índole social y cultural -incluidos los regímenes diferenciados de tenencia de la tierra, las tensiones familiares internas y los conflictos con las comunidades colindantes- explican, en buena parte, las trayectorias posteriores a la reforma agraria que llevaron a la disolución de esta empresa asociativa y de otras. 
El caso de la SAIS Picotani no es excepcional. En la provincia de Melgar, la ERPS Kunurana muestra lo recurrente de este problema $^{10}$. Así, los informes técnicos presentados por la Comisión Nacional de Propiedad Social del gobierno (CONAPS) en 1978 muestran una situación denominada por los propios funcionarios de la reforma como "asedio interno". Esta situación se caracteriza por la presencia de ganado huaccho (se calculó que existían 16779 cabezas en 1976 y 1977), cuando la mayoría de pastos de la exhacienda se limitaba a pasturas altas de baja calidad. El cuadro 2 muestra el recuento de ganado realizado en 1978 y da cuenta de un incremento de 1552 unidades de ganado huaccho en dos años, luego de conformada la empresa. Lo anterior, como anota Caballero (1978), estaría evidenciando que el plan del gobierno para reducir el ganado de los feudatarios no estaba dando resultado.

Cuadro 2. Cantidad y tipo deganado delaERPS Kunurana, 1978

\begin{tabular}{|c|c|c|}
\hline Tipo de ganado & $\begin{array}{c}\text { Ganado de la empresa } \\
\text { (marzo 1978) }\end{array}$ & $\begin{array}{c}\text { Ganado huaccho de } \\
\text { los socios (mayo 1978) }\end{array}$ \\
\hline Ovinos & 34841 & 4713 \\
Vacunos & 22496 & 7741 \\
Alpacuchos & 17850 & 3864 \\
Equinos & 360 & 2013 \\
\hline Total & 75547 & 18331 \\
\hline
\end{tabular}

Fuente: Informe de la ERPS Kunurana, recuperado por José María Caballero (1978).

10 La ERPS Kunurama, en la provincia de Melgar, comprendía 38956 hectáreas. Anteriormente, Kunurana había sido una SAIS perteneciente al PIAR -III (Umachiri-Nuñoa). La empresa ganadera se había conformada a través de la adjudicación de un conjunto de fundos expropiados en distintos momentos durante la década de 1970. En 1976, se le adjudicaron 14 nuevos predios rústicos con una extensión de veintinueve mil hectáreas y la Dirección Regional de Reforma Agraria la convirtió en una ERPS. 
La situación anterior se agravó si se considera la calidad y el tipo de pastos de la ERPS. Como muestra la figura 1, los pastos cultivados y el forrajero ocupaban una extensión mínima en el territorio de la Kunurana, lo cual hacía inviable la mejora de la productividad ganadera. Todo ello muestra un problema mayor: la presión por los pastos sumada a la incongruencia de dos lógicas distintas de manejo y tenencia. La permanencia de una lógica pastoril previa, que data de la época de la hacienda, no fue bien comprendida por el aparato de la reforma agraria que apostó por (i) la progresiva reducción del ganado familiar, (ii) el establecimiento de un vínculo puramente salarial con los feudatarios y (iii) el acceso regulado a los pastos a partir de una zonificación técnica de los mismos. Si bien esta última buscaba un mejor uso de las pasturas, rompía al mismo tiempo los antiguos sistemas de ocupación espacial y de rotación de pastizales atravesados por relaciones sociales que iban más allá de un arreglo contractual basado en una lógica de eficiencia. Así, por ejemplo, el pago por yerbaje impuesto por la empresa era percibido de manera negativa por los exfeudatarios socios de la ERPS. A esta discusión hay que agregar el peso y la tensión generada por las comunidades socias que quedaban como islotes en el interior de la empresa.

Retomando lo anterior, en 1978, la SAIS Picotani -a pesar de no tener pérdidas- y la ERPS Kunurana -que sí mostraba crisis financiera-, compartían tres situaciones contraproducentes: (i) la presión del ganado huaccho sobre los pastos y cientos de socios que mantenían miles de unidades de ganado de su propiedad fuera del manejo técnico de la empresa; (ii) los pastores exfeudatarios reproducían la lógica de uso de pastizales en las zonas altas para su ganado huaccho y mantenían relaciones de subordinación con los pastores que tenían menos acceso y menores recursos, con lo cual contradecían el principio básico de la empresa asociativa; y (iii) las comunidades socias no se llegaron a integrar al modelo, con lo cual 
la presión interna por tierras siguió latente. A ello se sumó la presión externa de las comunidades colindantes, tema que se aborda a continuación.

Figura 1. Calidad de pastos de la ERPS Kunurana (Informe CONAPS s/f)

\begin{tabular}{cccc}
\hline Clase Económica & $\begin{array}{c}\text { Syperficie } \\
\text { (has) }\end{array}$ & $\begin{array}{c}\text { Soportabilidad } \\
\text { por ha. }\end{array}$ & en u.o. total \\
\hline I & - & - & - \\
II & 8274.72 & 3 & 24854 \\
III & 5811.75 & 2 & 11624 \\
IV & 15483.14 & 1 & 15483 \\
V & 7404.39 & 0.6 & 4443 \\
Forrajes cultivados & 83.50 & 22 & 1837 \\
Tierras marginales & 1323.51 & - & - \\
Total & 38391.01 & - & 58241 \\
Total u.o de la & & - & 64738 \\
empresa & & - & 16779 \\
Total u.o huacchos & & & 23276 \\
Direfencia u.o $(-)$ & & & \\
\hline Fuente: Informe de CONAPS sin fecha & &
\end{tabular}

Fuente: Informe de CONAPS, citado en informes de José María Caballero (1978).

\section{Asedio externo: comunidades colindantes, tomas de tie- rra y procesos de comunalización}

Entre la adjudicación de tierras a las SAIS y las tomas de tierra en diversos lugares del departamento, transcurrió una década en la cual ni los niveles productivos de la SAIS mejoraron, ni las tensiones con las familias comuneras se apaciguaron. Como se analizó en la sección previa, la SAIS era frágil tanto en su estructura de funcionamiento como en la cohesión interna entre directivos, exfeudatarios y comunidades socias. Además, el resonar de las famosas tomas de tierra de las comunidades de Melgar hacia la ERPS Kunurana en 1985 y la consolidación de un movimiento campesino que había logrado posicionarse a nivel provincial y departamental (Rénique, 2004; Vega, 2000) generaban presión política a la 
Dirección Agraria Regional y, al mismo tiempo, respaldaba otros pedidos de repartos de tierra en Puno. La revisión de los archivos de la Dirección Regional de Reforma Agraria muestra que el caso de Picotani no fue la excepción; un conjunto de pedidos de reestructuración puede rastrearse en actas presentadas por las comunidades colindantes de diversas SAIS. Por su parte, Kunurana se convirtió en el caso emblemático de la reestructuración agraria en Puno y en el país por las masivas movilizaciones campesinas que reconfiguraron la estructura de la propiedad en el sur -con más de un millón de hectáreas redistribuidas- e impulsaron un proceso de comunalización que resultó en la formación de más de 500 nuevas comunidades campesinas (Rénique, 2004; Vega, 2005).

En el caso de Picotani, las comunidades indígenas de origen previo a la reforma, vecinas de la SAIS, habían quedado inconformes con la adjudicación de tierras realizada por el gobierno militar. Estas prácticamente no habían recibido tierras de los fundos expropiados y consideraban que las familias de exfeudatarios se habían beneficiado de manera desproporcionada. Aprovechando la coyuntura en el resto del departamento, y haciendo referencia al decreto de reestructuración agraria aprobado por el gobierno aprista en 1987, las comunidades decidieron presentar el expediente de pedidos de repartos de tierra a la administración de Picotani. El escenario que se configuró a partir de ello es revelador: las comunidades socias de la SAIS tomaron posición a favor de las comunidades colindantes, de acuerdo con el principio de que "las tierras deben ser para los hermanos campesinos que las necesitan", como consta en diversas actas de asambleas comunales. Entre 1985 y 1987, se realizaron numerosas asambleas convocadas por las comunidades de Huayllapata, Uyuni, Pistuni y Quilca Puncu, socias de Picotani, en las que discutieron durante horas y días la postura respecto a las tierras de la SAIS para finalmente acordar que apoyarían la entrega de tierras a las cuatro comunidades vecinas (Figura 2). 
A fines de 1987, se aprobó la resolución directorial para la transferencia de tierras de Picotani a favor de las comunidades campesinas de Cullco Belén, Coullco Jurinsaya, Chuquine y Llaulli. Así, se repartieron entre 350 y 22000 ha, dependiendo de la comunidad, ubicadas en los distritos de Muñuni y Putina, en la provincia de Azángaro. Se les cedieron varios fundos de las zonas altas, a más de 4200 m.s.n.m. y las comunidades se comprometieron a mantenerlos bajo régimen de tenencia colectiva, como se registra en el punto cuarto del acta de adjudicación a favor de la comunidad Culloc Belén (Figura 2) ${ }^{11}$. Sin embargo, al poco tiempo, las comunidades acordaron parcelarse internamente para adoptar un régimen de tenencia familiar, aunque siguieron manteniendo el régimen jurídico de comunidad campesina, que otorgaba protección al territorio comunal ${ }^{12}$.

11 Expediente de adjudicación de tierras de la SAIS Picotani a favor de comunidades (DRA-Puno-1988).

12 La propiedad comunal gozaba de la protección de sus tierras al ser consideradas como inalienables, imprescriptibles e inembargables por las constituciones de 1933 y 1979. Esto cambió en 1993, con la constitución neoliberal aprobada por el gobierno de Alberto Fujimori. 
Figura 2. Acta de adjudicación de la SAIS Picotani a la comunidad Cullco Belén (DRA- Puno-1988)

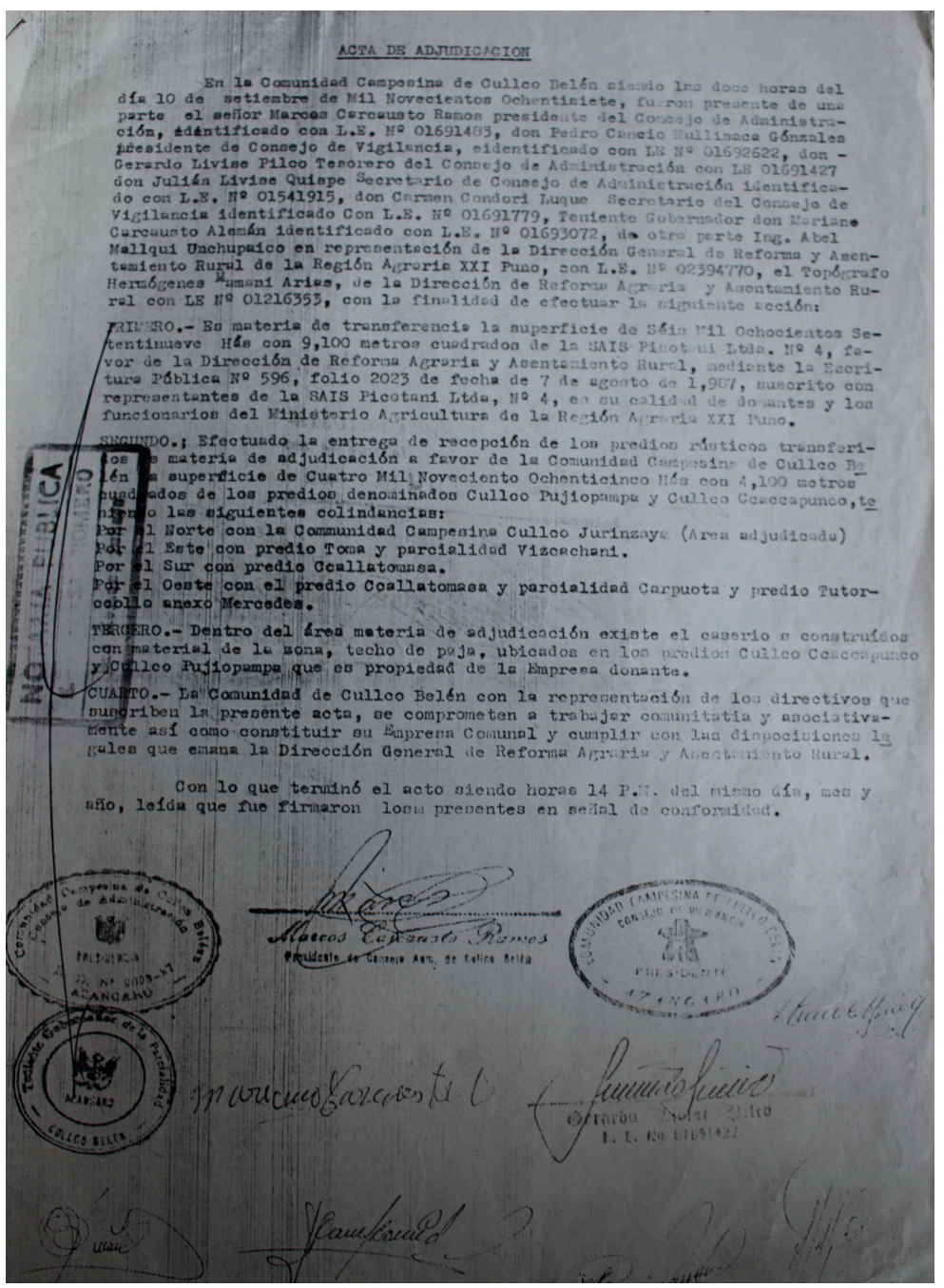

Uno de los aspectos sociales que merece unas líneas aparte es el de la postura de los comuneros en las propias SAIS. El 
caso Picotani ejemplifica este punto. En más de diez actas de asambleas ${ }^{13}$ constan las posturas de las comunidades socias respecto a los pedidos de tierras de las comunidades colindantes, como se transcribe a continuación:

Que la SAIS Picotani, especialmente los beneficiarios adjudicados de Reforma Agraria, están conscientes de que deben colaborar con los esfuerzos de entregar tierras a las comunidades, cooperando así con los trabajos de reestructuración de las empresas campesinas, para beneficiar a los hermanos comuneros ${ }^{14}$.

El registro anterior es significativo, ya que muestra el acuerdo, en un sentido político, sobre la disposición de las tierras de la SAIS y la situación injusta de la distribución y acceso a tierras de las comunidades no adjudicatarias. En este caso, a diferencia de la Kunurana, las tomas de tierra no fueron necesarias. La presión política de las comunidades socias contribuyó a hacer realidad la cesión de tierras de Picotani a favor de las comunidades colindantes. Luego de las primeras entregas de tierra, en 1992, la administración de Picotani aprobó la transferencia de 52236 hectáreas a la DRA-Puno, para que esta las transfiera a su vez a 21 comunidades y a un GAST $^{15}$, que solicitaba tierras desde años atrás. Así, las tierras de Picotani se fragmentaron a lo largo de una década y se aceleró la parcelación interna que llevó a su desaparición.

13 Estas actas están a escritas a mano y se encuentran fotocopiadas en los expedientes de adjudicación a las comunidades de la SAIS Picotani y transferencias de tierras a la D.G.R.A. y A.R. (DRA-Puno-1988).

14 Fragmento de asamblea comunal, SAIS Picotani 1987, registrada en el Expediente de transferencia de tierras rústicas en calidad de "donación" de la SAIS Picotani a la DRA-Puno para la reestructuración agraria. Folio de "Adjudicación a la comunidad campesina Chuquine de la SAIS Picotani” (DRA-Puno-1988).

15 Expediente de transferencia de tierras rústicas en calidad de "donación" de la SAIS Picotani a la DRA-Puno para la reestructuración agraria (1992). 
¿Cómo fue posible este resultado? La respuesta no es simple. Como se ha visto, la trayectoria hacia la comunalización y la parcelación se relaciona principalmente con la búsqueda de las familias campesinas de tener acceso directo a la tierra. Ello se hizo realidad mediante la suma de factores: por un lado, la capacidad de las dirigencias comunales -y sus asesores- para mostrar la desigual distribución de las tierras y, por otro lado, el respaldo del movimiento campesino que había realizado tomas de tierra por diversos lugares del departamento y que en esa época era capaz de movilizar a miles de familias comuneras. A lo anterior se sumó la situación interna de falta de cohesión de las propias SAIS, expresada parcialmente en la postura comunal y en el fragmento transcrito, pero también, como se ha mostrado en la sección previa, en las dinámicas de los pastores y la distancia entre el manejo empresarial del ganado y el uso de los pastos que transgredía, a todas luces, los intentos de zonificación de los mismos. Estos fueron realizados con criterios técnicos, que excluyeron consideraciones históricas y culturales de la apropiación de dicho territorio.

Por los mismos años, en otro ámbito del departamento, en la zona de Melgar, se gestaba el más grande proceso de reestructuración agraria a nivel nacional. En ese caso, mediado a través de la acción política del movimiento campesino y la estrategia de las tomas de tierra. Las comunidades campesinas de ese ámbito, como Macarí y Santa Rosa, desde los años de la formación de la ERPS Kunurana $(1976)^{16}$, demandaban al gobierno la reestructuración de las tierras de la empresa a favor de las comunidades que no habían sido beneficiarias de la reforma agraria. Para ello, las comunidades habían trabajado con organismos de desarrollo, como el Centro de Capacitación Campesina de Puno (CCCP), dirigido por Ricardo Vega. En un proceso de casi diez años, las comunidades ha-

16 Expedientes del proyecto de transferencia de predios rústicos a la ERPS Kunurana. DRA-Puno $(1976,1977,1980)$. 
bían formulado pedidos a la Dirección Regional de Reforma Agraria de Puno y al Ministerio de Agricultura en Lima, para lo cual habían elaborado un expediente de reestructuración en el que constaban las diferencias en la distribución de tierras, como se muestra en el cuadro 3. Además del problema de la cantidad de tierra, las ocho comunidades colindantes de la ERPS argumentaban que la empresa había concentrado las mejores tierras, mientras que las comunidades tenían un gran porcentaje de sus territorios en zonas eriazas, tierras arcillosas y laderas rocosas de los cerros.

\section{Cuadro 3. Estructura y tenencia de la tierra en la ERPS Kunurana y las comunidades colindantes antes de la reestructuración (Melgar, Puno)}

\begin{tabular}{|l|c|c|c|}
\hline $\begin{array}{c}\text { Comunidad } \\
\text { campesina }\end{array}$ & $\begin{array}{c}\text { Cantidad de } \\
\text { hectáreas }\end{array}$ & $\begin{array}{c}\text { Número de } \\
\text { familias }\end{array}$ & $\begin{array}{c}\text { Promedio de } \\
\text { hectáreas/ } \\
\text { familia }\end{array}$ \\
\hline Macarí & 753 & 120 & 6.2 \\
\hline Selque & 7322 & 106 & 69 \\
\hline Huamanruro & 3688 & 223 & 16.5 \\
\hline Quishuara & 7140 & 376 & 19 \\
\hline Santa Rosa & 544 & 65 & 8.4 \\
\hline Kunurana alto & 4190 & 101 & 41.5 \\
\hline Kunurana bajo & 4960 & 100 & 49.6 \\
\hline Picchu & 433 & 59 & 7.3 \\
\hline $\begin{array}{l}\text { Total de ha 8 } \\
\text { C.C. }\end{array}$ & 29030 & 1150 & --- \\
\hline ERPS Kunurana & 38956 & 154 & 253 \\
\hline
\end{tabular}

Fuente: Expediente del Comité Técnico de Reestructuración (Elaboración: R. Vega, 2005, p. 66).

Además del expediente técnico de reestructuración -elaborado inicialmente para solicitar tierras a favor de la comunidad 
indígena Macarí-, es posible comprobar en otras fuentes la diferencia en el acceso a pastos entre los exfeudatarios, socios de la ERPS y las familias de las comunidades vecinas ${ }^{17}$. Así, por ejemplo, el atlas estadístico microrregional de 1977 seńala que Kunurana contaba con 90962 cabezas de ganado, 405 hectáreas de pastos cultivados y más de 38000 hectáreas de pastos naturales, mientras que las ocho comunidades colindantes sumaban un total de 66 hectáreas de pastos instalados y unas 29000 hectáreas de pastos naturales y zonas eriazas ${ }^{18}$.

En 1981, la elaboración del expediente técnico venía de la mano con un proceso organizativo: se conformó el comité técnico para la reestructuración democrática, que incluyó a los asesores técnicos y a representantes de las comunidades de la zona, así como a dirigentes de la Federación Unitaria de Campesinos de Melgar (FUCAM), como Julián Páucar, y de la Federación Departamental de Campesinos de Puno $(\mathrm{FDCP})^{19}$. Entre 1981 y 1985, hubo diversos pedidos de reestructuración, que no fueron atendidos por el gobierno. En 1983, el informe técnico fue presentado a CORPUNO y en 1984 al Ministerio de Agricultura. Sin embargo, las federaciones campesinas nacional, departamental y provincial no tuvieron respuesta. Ante la reiterada negativa de la Dirección Regional Agraria y los vanos intentos de llegar al ejecutivo nacional, en una gran asamblea campesina con la presencia de todas las comunidades de Melgar, de dirigentes de la

17 CCP-FDCP “Reestructuración democrática del agro puneño. Vía campesinas comunera" (1986, p. 8).

18 Atlas estadístico de las micro regiones del departamento de Puno - Oficina de Planificación Agraria, 1977.

19 Creada en 1978, la Federación Departamental de Campesinos de Puno fue cercana a Vanguardia Revolucionaria entre 1978 y 1985, año de las tomas de tierra de Melgar, y luego al Partido Unificado Mariateguista (PUM) en los años 1985-1993. (Entrevista a Julián Páucar, dirigente de la FUCAM y la FDCP). 
FUCAM y de la Confederación Campesina del Perú se decidió la toma de tierras del Fundo Unión Milloni, propiedad de la ERPS Kunurana ${ }^{20}$.

Así, luego de casi una década de pedidos y negociaciones infructuosas, el 13 de diciembre de 1985 se movilizaron miles de comuneros para tomar 10000 hectáreas de tierras de $\mathrm{Ku}$ nurana, como relata el entonces presidente de la FUCAM: "fueron días de lucha, llegaron miles de comuneros de todos los sitios, marchamos para tomar las tierras del fundo Unión Milloni [perteneciente a Kunurana], iban llegando a pie, en camiones, así ha sido" ${ }^{21}$.

Los eventos descritos permiten argumentar que las tomas de tierra por las comunidades de Melgar fueron el resultado de un proceso de debate y movilización, y no una acción improvisada. Luego de estas primeras tomas, el gobierno aprista aprobó una primera reestructuración de la ERPS, pero las trabas del proceso terminaron por desencadenar las tomas de tierra más grandes del país. Los dirigentes de la FDCP y de la FUCAM la catalogaron como una reestructuración fraudulenta, una estrategia para apaciguar a las comunidades. Como expresa el fragmento siguiente rescatado por Vega, las comunidades consideraban que se trataba de "una reestructuración autoritaria, antidemocrática y antitécnica, sin participación de las comunidades campesinas ni la FDCP..." (Vega, 2005, p. 90). Asimismo, denunciaban que a las comunidades se les había entregado nuevas tierras, pero en zonas muy alejadas e incluso con superposición de beneficiarios (dos comunidades con una misma zona adjudicada), lo cual

20 Para comprender las federaciones campesinas, la presencia de los partidos de la llamada nueva izquierda y la Iglesia católica a través de las vicarías del sur andino, véase Rénique, 2004.

21 Entrevista a Julián Páucar, actual presidente de la FDCP y expresidente de la FUCAM durante las tomas de tierra en Melgar en 1985 y 1987. 
generó problemas de linderos, que es posible rastrear hasta la actualidad. Esta situación llevo en 1987 a una movilización de alcance departamental. Se calcula que unas 15000 familias de 300 comunidades campesinas tomaron 640000 hectáreas de diversas SAIS y ERPS en todo Puno, entre mayo y junio. Luego de ello, el gobierno aprista se vio en la necesidad de emitir el Decreto Supremo 006-1987, "aprobando" la reestructuración de las tierras de las empresas asociativas del departamento en beneficio de las comunidades campesinas.

El resultado de este proceso reacomodó la estructura de la propiedad en Puno: de 1740311 hectáreas adjudicadas a las 42 empresas asociativas entre 1973 y 1976, las comunidades se reapropiarían de 1010992 hectáreas durante el período de la reestructuración. Con ello, la comunidad campesina se consolidó como una organización y como instancia de decisión colectiva. Se formalizaron e inscribieron alrededor de 500 nuevas comunidades en el departamento entre 1985 y 1990. En estas, como es sabido, el régimen de tenencia fue el de parcelas individuales administradas directamente por las familias comuneras, en coexistencia con ciertas áreas de tenencia mixta o de manejo comunal de pastos con variantes entre comunidades (Burneo y Trelles, 2019). Durante la década de 1980, entre comunidades originarias que no gozaban de inscripción previa y comunidades conformadas a raíz de la reorganización de las tierras, se inscribieron oficialmente 788 comunidades campesinas, que agregadas a las existentes sumaban 1274 en $1994^{22}$.

Las trayectorias posteriores a la reforma agraria en el sur andino tuvieron, por tanto, como principal característica, un proceso de comunalización como estrategia de las familias

22 Archivos internos proporcionados en formato digital (Excel) para esta investigación por la Dirección Agraria Regional de Puno en julio de 2018. 
campesinas para garantizar su acceso a la tierra con reconocimiento estatal, un régimen variable de tenencia en su interior y una clara tendencia hacia la tenencia familiar o individual. Como han evidenciado Burneo y Trelles (2019) para el caso de Ilave y Azángaro en el mismo departamento, entre 1992 y 1994 -y en adelante-, muchas de estas comunidades siguieron el camino hacia la parcelación ${ }^{23}$. Es decir, un régimen comunero pero basado en la microocupación familiar del espacio, en el que la comunidad se transformó y redefinió sus funciones, pero permaneció como un espacio de decisión en aspectos colectivos que confluyeron en la década de los años noventa con nuevos agentes políticos e institucionales -como las municipalidades de centros poblados, las rondas campesinas y los tenientes gobernadores-, que paulatinamente fueron cobrando mayor peso en la nueva configuración del poder local.

\section{Conclusiones}

Las grandes empresas asociativas formadas durante la reforma agraria en Puno, al igual que en otras regiones, siguieron un camino marcado por presiones internas y externas que las llevaron a su parcelación y disolución, con muy pocas excepciones en el país. En este departamento, la trayectoria regional lo llevó hacia un proceso de comunalización, similar

23 Entre los factores que explican la parcelación, se puede mencionar el proceso de municipalización (Quiñones, 2011), el abandono progresivo de las comunidades y la pequeña agricultura familiar por parte del Estado (Eguren, 2004) y nuevos flujos migratorios (Burneo y Trelles, 2019; Pajuelo, 2005; Vilca, 2015), entre otros cambios. A pesar de ello, a fines de 2018 existían 1303 comunidades campesinas inscritas en el departamento, propietarias de más de dos millones de hectáreas de tierra tituladas, es decir, la tercera parte de todo Puno. 
al del centro (Junín), pero con la particularidad de que se consolidó un régimen de apropiación individual que coexistió con una instancia colectiva. Las trayectorias presentadas a partir del caso de la SAIS Picotani y la ERPS Kunurana muestran inconsistencias del modelo del agro asociativo, que van más allá del manejo administrativo y financiero, y relevan el peso de la dimensión histórica y social. Estas suponen lógicas y patrones diferenciados de usos del espacio y de los recursos que se combinan con acuerdos previos, cuyas huellas no fueron fáciles de borrar, como tal vez asumieron algunos cuadros técnicos del aparato reformista. Tanto los informes técnicos revisados, como los archivos de actas de asambleas comunales y las entrevistas realizadas a actores clave de esta historia en Puno plantean la importancia de dos procesos paralelos: la presión interna por recursos -principalmente pastos-, y la presión de las comunidades vecinas, cuyas expectativas por el acceso a más y mejores tierras no se había visto cubierta con las adjudicaciones de la década de 1970 . Ambos procesos definieron buena parte de las trayectorias posteriores de las empresas asociativas en el departamento.

Los casos de Picotani y Kunurana dan cuenta de las limitaciones de un cambio apresurado e impuesto sobre los regímenes de tenencia de los pastores de altura. La nueva zonificación de pastos emprendida por la Dirección Regional de Reforma Agraria no tuvo los efectos esperados en un territorio, donde los huacchilleros circulaban en extensiones de pasturas de forma estacional. Por otra parte, la apuesta ideológica -sustentada en jornadas de capacitación y concientización dirigidas a los exfeudatarios-, no bastó para romper una lógica previa de manejo agropastoril ni para que las familias abandonaran su ganado familiar en beneficio del ganado de la empresa. El "asedio interno", como lo llama Caballero (1978), no se superó. Una escasa comprensión del peso de las lógicas previas y la ausencia de una lectura diacrónica constituyeron un error. Si bien este no explica por sí solo el 
fracaso del modelo, en el caso del sur andino sí se convirtió en una carga que las grandes empresas asociativas arrastraron como un lastre hasta su desintegración.

Los casos estudiados muestran también que es fundamental tomar en cuenta la configuración regional particular: el peso de las comunidades y la confluencia de un conjunto de actores que van desde la ONG de desarrollo rural, partidos de la nueva izquierda, federaciones campesinas (FCDP y FUCAM) y hasta la apuesta de la Iglesia católica surandina por la vía comunera, que le otorgaron un sustento institucional fuerte al proceso de la reestructuración. Este se dio por la vía de las tomas de tierra, pero tenía como base acciones previas tanto técnicas como jurídicas. A lo anterior se sumó que en Puno Sendero Luminoso no logró tener el impacto que tuvo en la destrucción parcial de SAIS, como la Cahuide en Junín, entre otras (Comisión de la Verdad, 2003). Así, la tan discutida vía comunera (Rénique, 2004, p. 284; Vega, 2005), finalmente, terminó siendo una apuesta distinta por la recuperación no solo de las tierras, sino de un proyecto comunal de reorganización del espacio y del poder local.

Todo ello, enfatiza la importancia que tuvieron los procesos sociales -de origen previo a la reforma y que fueron más allá de ella- en las transformaciones rurales del espacio sur andino. Dentro de esos procesos, se consideran especialmente: (i) la movilización de las familias comuneras por la administración directa de la tierra, (ii) la presencia de comunidades (algunas de ellas consideradas ancestrales por su origen previo a la llegada de las haciendas) y (iii) los nuevos procesos de comunalización derivados de las tomas de tierras y la reestructuración agraria. En suma, los efectos de la reforma agraria a mediano plazo en el caso de Puno permitieron mayor acceso a las tierras para las familias campesinas y comunidades. Es importante seńalar que esto no quiere decir que todas ellas sean de origen reciente; un grupo de estas comunidades -no 
es posible señalar la cifra exacta- en efecto lo son, ya que se formaron de nuevos grupos campesinos conformados durante la reforma, pero otras son antiguas comunidades que habían sido parcialmente absorbidas o habían quedado en la periferia de las haciendas y lograron su inscripción luego de la reestructuración y de la aprobación de la Ley General de Comunidades de 1987; otras, finalmente, provienen de antiguas parcialidades indígenas -que aún hablan de sus ayllus de procedencia- y se juntan para formar una sola comunidad, logrando así reconocimiento y protección estatales.

Lo anterior permite concluir que las trayectorias regionales de la reforma agraria -que incorporaron microprocesos previos-, contribuyeron a trascender una lectura de esta como un evento puntual que culminó con la caída del modelo del agro asociativo, para enmarcarla como parte de un proceso de mayor alcance en el que se traslaparon proyectos distintos -como la recuperación campesina de las tierras-, que tomaron una forma o rumbo particular a partir de ella. Más allá de la desintegración de las grandes SAIS, lo cierto es que la reforma agraria en su trayectoria regional particular abrió paso a la reestructuración de más de un millón de hectáreas y a un proceso de comunalización con una forma de ocupación familiar de la tierra. Luego de ello, el camino seguido por un número importante de comunidades fue el de la parcelación de las tierras entre sus comuneros, manteniendo sin embargo la organización comunal como espacio de gestión y toma de decisiones colectivas.

Recibido: 15 de noviembre de 2019

Aprobado: 20 de marzo de 2020 


\section{Referencias bibliográficas}

ÁVILA, Y.

(1977) La deuda agraria en Puno: el carácter corporativo del "no pago de la deuda agraria". Lima: Ediciones José María Arguedas.

BOURRICAUD, F.

(2012) Cambios en Puno: estudios de sociología andina. Lima: Instituto de Estudios Peruanos.

BURNEO, M.

(2019) Reforma agraria desde abajo. Lucha por la tierra, excooperativistas y parcelación entre los comuneros de Catacaos, Piura. Argumentos Revista de Análisis y Crítica, 13 (2), 19-29. Recuperado de https://argumentos-historico.iep.org.pe/articulos/la-reformaagraria-desde-lucha-la-tierra-excooperativistas-parcelacion-los-comuneros-catacaos-piura/

BURNEO, M. y A. TRELLES

(2019) Nueva ruralidad y comunidades en Puno. En P. Vilca (Ed.). Puno en el siglo XXI: Desarrollo, ambiente y comunidad (pp. 182-234). Lima: Asociación SER.

CABALLERO, J. M.

(1978) Informes inéditos del estudio "Reforma y reestructuración agraria". Custodiados por el Archivo documental de Instituto del Perú, de la Universidad San Martín de Porres. Lima: Universidad San Martín de Porres.

CABALLERO, J. M. y ÁLVAREZ, E.

(1980) Aspectos cuantitativos de la reforma agraria. Lima: Instituto de Estudios Peruanos. 
CABALLERO, $\mathrm{V}$.

(1990) Cambios en la propiedad de la tierra: Estudio de la SAIS Cahuide y las comunidades socias. En Perú, el Problema Agrario en Debate. Seminario Permanente de Investigación Agraria, III. Cusco: SEPIA.

CASTILLO, P. del

(2013) IV CENAGRO y las comunidades del país; su presencia es innegable. La Revista Agraria, 12-13.

CHE PIU SALAZAR, A.

(1976) Acerca de la problemática de los huacchos. En: Puno: Problemas campesinos (1), 55-64. Puno: IDISA.

COMISIÓN DE LA VERDAD Y LA RECONCILIACIÓN (2003) Informe final. Lima, CVR.

DAMONTE, G. y RODRÍGUEZ, S.

(2016) La evolución de los regimenes de tenencia colectiva de la tierra en sociedades pastoriles. Lima: GRADE. Serie Documentos de Investigación ELLA.

DIEZ, A.

(2007) Organización y poder en comunidades, rondas campesinas y municipios. En A. D. P. Castillo, ¿Qué sabemos de las comunidades campesinas? (pp. 108152). Lima: ALLPA.

DIEZ, A.

(2017) Propiedad y territorio como (diferentes) bienes comunes. El caos de las tierras de comunidades en la costa norte peruana. Eutopía (11), 17-39. https:// doi.org/10.17141/eutopia.11.2017.2851

DIEZ, A. y M. BURNEO

(2019) Agro asociativo y procesos sociales: Transformaciones rurales desde la reforma agraria en la sierra centro y sur peruana. Ponencia presentada en el Seminario Bianual Permanente de Investigación Agraria, XVIII. Puno: SEPIA. 
EGUREN, F.

(1988) La reforma agraria y el nuevo orden del campesino peruano. Santiago de Chile: Grupo de investigaciones agrarias. Comisión de Estudios Rurales.

EGUREN, F.

(2004) Las políticas agrarias en la última década: una evaluación En: Perú, el Problema Agrario en Debate. Seminario Permanente de Investigación Agraria, X. Lima: SEPIA.

FAVRE, $\mathrm{H}$.

(1976)

Evolución y situación de la hacienda tradicional en la región de Huancavelica. En: J. Matos Mar (Comp.), Hacienda, campesinado y comunidad (pp. 105-138). Lima: Instituto de Estudios Peruanos.

GASCÓN, J.

El control y explotación de la mano de obra colona en la hacienda andina peruana. Anuario de Estudios Americanos, 56 (1), 195- 215. https://doi. org/10.3989/aeamer.1999.v56.i1.293

GASCÓN, J.

(2017) Acabando con la administración de poblaciones. Razones y estrategias para reclamar la plena ciudadanía en la hacienda surandina peruana. En V. Breton y M. J. Vilalta (Eds.), Poderes y personas: pasado y presente de la administración de poblaciones en América Latina (pp. 195-216). Barcelona: Icaria Editorial.

INSTITUTO DE INVESTIGACIONES SOCIALES DEL ALTIPLANO

(1976)

Puno: Problemas campesinos. Documento de trabajo, n. ${ }^{\circ}$ 1. Puno: IDISA.

JACOBSEN, N.

(1993) Mirages of Transition. The Peruvian Altiplano, 17801930. Berkeley: University of California Press. https://doi.org/10.1525/9780520913912 
LONG, N. y ROBERTS, B.

(1978) Peasant cooperation and capitalist expansion in Central Peru. Austin: University of Texas.

MARTÍNEZ ALIER, J.

(1973) Los huacchilleros del Perú: dos estudios de formaciones sociales agrarias. Lima: Instituto de Estudios Peruanos, Ruedo Ibérico.

MATOS MAR, J. y MEJÍA, J.

(1980) La reforma agraria peruana. Lima: Instituto de Estudios Peruanos.

MAYER, E.

(2009) Cuentos feos de la reforma agraria. Lima: Instituto de Estudios Peruanos.

PAJUELO, R.

(2009) No hay ley para nosotros. Gobierno local, sociedad y conflicto en el altiplano: el caso Ilave. Lima: Instituto de Estudios Peruanos.

PLOEG, J. W. van der

(2018) The New Peasentries. Struggles for Autonomy and Sustainability in an Era of Empire and Globalization. London and Sterling: Earthscan Publications Ltd.

POZO-VERGNES, E. del

(2004) De la hacienda a la mundialización: sociedad, pastores y cambios en el altiplano peruano. Lima: IFEA, Instituto de Estudios Peruanos. https://doi.org/10.4000/ books.ifea. 4803

QUIÑNONES, P.

(2011) Municipalidades distritales rurales del Altiplano: los nudos de la política local. Lima: SER. 
REMY, M.

(1976) Proceso y límites de la proletarización en Laive. En G. Rochabrún, B. Maza, M. I. Remy y M. G. Vega, Campesinado y capitalismo en el Perú: aproximaciones a partir de la economía politica. Lima: Pontificia Universidad Católica del Perú, Departamento Académico de Ciencias Sociales.

RÉNIQUE, J.

(2004) La batalla por Puno: Conflicto agrario y nación en los Andes peruanos 1866-1995. Lima: Instituto de Estudios Peruanos.

REVESZ, B.

Necesidad de una nueva interpretación de la reforma agraria y sus aspectos, estructura agraria y formas de producción asociativas: balance y perspectivas de la investigación reciente. En Perú: El Problema Agrario en Debate, Seminario Permanente de Investigación Agraria, I (pp. 87-122). Lima: SEPIA.

SCURRAH, M.

(1987) Empresas asociativas y comunidades: Puno después de la reforma agraria. Puno: Grupo de Estudios para el Desarrollo GREDES.

TRIVELLI, C.

(1992) Reconocimiento legal de comunidades campesinas: una revisión estadistica. Lima: CEPES.

VEGA, R. (2005) La lucha por la tierra en Puno. Puno: Centro de Capacitación Campesina de Puno (CCCP).

VILCA, P.

(2015) La persistencia de la politica: redes politicas en el altiplano puneño (Tesis para optar el grado de magíster en ciencias políticas). Pontificia Universidad Católica del Perú, Departamento Académico de Ciencias Sociales, Lima. 